\title{
Some Remarks on the Physicalist Account of Mathematics
}

\author{
Ferenc Csatári \\ Department of Logic, Institute of Philosophy, Eötvös Loránd University, Budapest, Hungary \\ Email: fcsatari@gmail.com
}

Received February 28 ${ }^{\text {th }}$, 2012; revised March 25 ${ }^{\text {th }}$, 2012; accepted April $9^{\text {th }}, 2012$

\begin{abstract}
The paper comments on a rather uncommon approach to mathematics called physicalist formalism. According to this view, the formal systems mathematicians concern with are nothing more and nothing less than genuine physical systems. I give a brief review on the main theses, then I provide some arguments, concerning mostly with the practice of mathematics and the uniqueness of formal systems, aiming to show the implausibility of this radical view.
\end{abstract}

Keywords: Physicalism; Formalism; Philosophy of Mathematics

\section{Introduction}

On the following pages I comment on a rather uncommon philosophical approach to mathematics called physicalist formalism. ${ }^{1}$ I give a brief review of the main theses, then I try to outline some of the main flaws of this approach. I am far from the intent to be exhaustive in this respect, as I am far from a consequent defence of an alternative approach. But I am convinced that the few arguments given here, concerning mostly with the practice of mathematics and the uniqueness of formal systems, are enough to show the implausibility of this radical view.

\section{Mathematical Assertions Have No Meaning}

The formalist-physicalist philosophy of mathematics lies on the empiricist extreme of the "ideologies" concerning mathematics. As a genuine empiricist view, it has its own questions and problems to cope with by its own way. As Ayer put it in this often recited paragraph:

"For whereas a scientific generalization is readily admitted to be fallible, the truths of mathematics and logic appear to everyone to be necessary and certain. But if empiricism is correct no proposition which has a factual content can be necessary or certain. Accordingly the empiricist must deal with the truths of logic and mathematics in one of the following ways: he must say either that they are not necessary truths, in which case he must account for the universal conviction that they are; or he must say that they have no factual content, and then he must explain how a proposition which is empty of all factual content can be true and useful and surprising. [… If neither of these courses proves satisfactory, we shall be obliged to give way to rationalism. We shall be obliged to admit that there are some truths about the world which we can know independently of experience."2

Physicalism, as it is outlined in Szabó's works, has to deal with both kinds of challenge. On one hand, mathematics is said to be an inductive science, so it cries for explanation why its truths (in a pre-theoretic sense) are usually thought to be necessary or

\footnotetext{
${ }^{1}$ See the works of Szabó L. E. (2003, 2009, 2010). Since the third one is a lecture note, I will refer to the first two as long it is possible.

${ }^{2}$ Ayer (1936), pp. 72-73.
}

its "laws" to be more solid than that of physics. ${ }^{3}$ On the other hand, physicalism asserts that mathematical statements do have factual content, though far not in the usually preached sense. So the urge for the explanation of useful and surprising results also remains. Szabó is up to accomplishing both of these tasks, but, I will argue, the results are unsatisfactory.

According to the physicalist-formalist a mathematical statement (a theorem) is true if and only if it can be derived from the given axioms. The truth-condition of a statement lies in the existence if its derivability. It is meaningless to talk about mathematical truth generally, only in accordance with a given axiom system. True, there have not always been reliable axiom systems, but that is only a contingent historical fact, and as such, lies out of mathematics. Many proofs worked out by the mathematicians of the past are of historical interest only, and not regarded as strict, genuine proofs today.

Szabó (2003) distinguishes two different senses of truth. The first kind is the truth of the theorems, i.e. the mathematical truth in a given formal system. The second kind of truth is the empirical truth, where a sentence refers to some empirical fact through some semantics. Now the main formalist thesis of the physicalist is this: mathematical objects and propositions have no meaning, they are only meaningless marks on the paper manipulated by some rules. Formulas do not carry Tarskian truth, they can be true in the first sense of truth only. The argument says that if a mathematical proposition were a statement of the factual world, the mathematician should carry out experiments as the physicist does. That is clearly not the case (especially if thought experiment is regarded as a special kind of non-experiment). ${ }^{4}$ Moreover, even if one does associate meaning to mathematical propositions, it is irrelevant to the truth of the given statement. Even if one ascribes truth of the second type to the axioms of some formal system, it is not

\footnotetext{
${ }^{3}$ Once I talked about Russell's thought experiment (in Russell (1912)) to one of my friends, an immaculate in philosophy. "Can you imagine that there are immortal humans?"-I asked. "Yes, of course I can”- he said. "But can you imagine that $2 \times 2$ is 5?" After a while he replied: "Yes, I can, but it makes me rather angry"-indeed, in a more informal way cannot be literally cited here.

${ }^{4}$ There is a bit of muddle here, however. Following the physicalist, as we will see, one would wonder what non-physicalist methods mathematicians could have.
} 
granted that it will be come down to the theorems derived from the axioms. In other words, whether a theorem derived from semantically true axioms is semantically true or not is a contingent fact of the world (would there be semantically true axioms at all). Maybe there are mathematical intuitions of which we have perceptions the same way we have perceptions of the physical world, ${ }^{5}$ but the truth mathematical statements concern with is independent of them. Note that this account is not simply a rewording of the old thesis: all mathematical statements are tautologies, i.e. analytical truths. As we will see in a moment, we cannot have analytical knowledge-simply because we cannot have a priori knowledge whatsoever.

The success of mathematics in sciences (and above all, in physics) is often considered as a strong argument for that it does embrace some crucial structures in the "real world". Moreover (as the so called Quine-Putnam-argument suggests), mathematics is indispensable in physics, and we have to have ontological commitments toward the indispensable. Szabó claims that this argumentation can be easily undermined. First, mathematics has much more than what is needed in physics. Second, reliable predictions are not made by mathematics itself, but by a complex physical theory with its semantics (of course, mathematical statements do not carry Tarskian truth). Third, there are more than one mathematical theories applicable in, say, physical predictions, and, forth, the appropriate mathematical theory is chosen by the physicists, not by external constraints.

Since mathematical theories (in themselves) are nothing more than formal systems, it is meaningless to talk about intended interpretations, standard models, intuitive arithmetic or set theory and so on. True, however, says the physicalist, that the axiom systems may not be entirely arbitrary: usefulness can be a criterion for choosing them. Anyhow, systems taken as "formal", , and among them the ones with interest for mathematicians are chosen along convention or usefulness-factors lying outside of mathematics. I am not sure how conventional and useful Peano-arithmetic is, but seemingly the physicalist does not take great pains to explain why some formal systems are interesting for mathematicians, why not others.

\section{There Are No Structures to Be Represented}

After the semantic apologetics for formalism, Szabó turns to the main genuine physicalist theses as the results of some ontological considerations. Though mathematical propositions have no meaning, they express objective facts about the formal systems they stem from. To be more precise: they express facts about the particular signs constituting the given system and the rules by which they are combined. Rules are also laid down by signs. The ontology of formal systems is thus so simple: there are only physical objects (signs) as participants in (contingent) physical facts.

The "physicality" of formal systems has two sides. First, the only

\footnotetext{
${ }^{5}$ As Gödel puts it in his defence of platonism: “[T]here is nothing in the least absurd in the existence of totalities containing members, which can be described (i.e., uniquely characterized) only by reference to this totality. [...] Classes and concepts may [...] be conceived as real objects [...] existing independently of us and our definitions and constructions. It seems to me that the assumption of such objects is quite as legitimate as the assumption of physical bodies and there is quite as much reason to believe in their existence.” Gödel (1944), p. 456.

${ }^{6}$ According to Szabó it is mere convention that the rules and "symbols" of chess are not regarded as an axiomatic system.
}

way for one to be informed of the truth of some mathematical fact is through a physical process. Of course, physical processes are required for one to be "informed" of anything — let them be historical speculations, artistic impressions or astrological constellations. Doing mathematics in the head or divine revelations are not exceptions - in a physicalist account there is always an underlying neurophysical process. Now, the knowledge of the truth of some mathematical proposition is a truth-condition of the given proposition. But how about other propositions? I mean, according to the physicalist, is the knowledge of the truth of the sentence: "It was a wild and stormy night on the West Coast of Scotland"7 a truth-conditions for it? If not, what the special status of mathematical statements lies in?

Second, mathematical propositions (the true ones, i.e. which can be derived) express objective facts of the physical world of the system. Thus, says Szabó with irony, we do have the ontological commitment that the Quine-Putnam indispensability argument claims for: mathematical statements "talk" about formal systems, i.e. strings of symbols, and we do believe in the existence of these entities.

It must be clear that the formal systems so understood are particular, concrete systems. There are no abstract things, abstract structures represented by them. It would be a categorical mistake to assume various "isomorphisms". Mathematical knowledge is not conventional (though choosing the particular topic (i.e. system) is), not perfect, not a priori, not certain. Mathematical truths reveal "contingent facts about a particular part of the physical world”. 8

Probably the most striking assertion of the physicalist is that there is no certain knowledge gained through deduction, since the latter is only a special case of induction-namely the one dealing with the facts of formal-physical systems. This claim is uncommon though, it follows from what is said so far. If the truth-condition for a mathematical proposition is nothing more than the knowledge of the given propositions, then there is no difference between knowledge and truth in this case. Formal systems are particular physical systems of which we can gain information by observations-thus induction.

True, mathematical truths appear necessary and certain truths for many. The reason is that formal systems usually have stable behavior among the physical systems and do not require "external" observations. But since "observations" and "(thought) experiments" provide the only way to get in possession of mathematical truths the certainty so gained is exactly the certainty of physical truths. According to the physicalist's consolation, the "certainty available in inductive generalization is the best of all certainties". 9

\section{Scientific Practice Does Not Belong to Mathematics}

One of the main morals of the physicalist approach is, I think, that mathematics is not what we usually mean when talking about "mathematics", be the concept however vague. Of course, rational reconstructions often require the revision of commonly used concepts for the sake of exactness. But I regard such a radical change in the concept of mathematics unjustified. Moreover,

\footnotetext{
${ }^{7}$ “ $[\ldots]$ This, however, is immaterial to the present story, as the scene is not laid in the West of Scotland. For the matter of that the weather was just as bad on the East coast of Ireland.” Leacock (1911), p. 45.

${ }^{8}$ Szabó (2009), p. 9.

${ }^{9}$ Szabó (2003), p. 10.
} 
my taste draws me to approaches with more bias toward scientific practice: as philosophical concerns can be of crucial importance for the science, philosophy likewise should not be blind for the actual scientific practice.

True, Szabó does mention the practice of mathematics:

"Of course, very much depends on how we understand the practice of the mathematician. I think, that in the same way like ordinary people who dream about movie stars but live with their partner, mathematicians rave about various platonic objects, but if they are seriously asked what they are confident about, they reduce their claims to mere if-thenisms. All the rest is just "folklore". And this holds not only for the more complex branches of mathematics but also for arithmetic and set theory. When the number theorist says "there are infinitely many prime numbers", or simply "7 is a prime number lager than 5" then (s)he means that all these concepts as "larger" and "prime", etc., are defined with formal rigor, and that the statement in question is a theorem within the corresponding formal framework. This is true even if (s)he proves it by simple calculations, since it was previously proved that the employed algorithm is correct. [… Thus, concerning the rigorous, scientifically justified, nonfolkloristic part of the claims of mathematicians, it is far from "unquestionable" that they are committed to something more than if-thenism." 10

Maybe I understand scientific practice in a different way. When a mathematician is working on a given problem always uses a language somewhat similar to the ordinary one, and, of course uses the tool of formal derivation in the given formal system. But the latter alone, isolated from the sloppy words and thoughts would hardly "work" as mathematics, we would have only ink-marks on a piece of paper, exactly as the formalist insists (I will address this point below). Maybe mathematicians are "Sunday-formalists", 11 but they do their work on weekdays. Undeniably, formal methods are of extreme importance for them, but other things have role in their scientific practice as well. Even when working with their pencil and paper, mathematician quite rarely do something like deriving proposition from a bulk of axioms.

I do not intend to inquire into what is in the mathematician's head. Assessing what she believes in, what she is committed to is not an easy mission. A much more modest project is just to regard what she does when doing mathematics. I am convinced that a philosopher dealing with mathematics should always keep one eye on this.

\section{Historical, Psychological Curiosities Do Not Belong to Mathematics}

It is easy to agree with this statement. However, glancing at mathematics from historical or psychological perspective could have some morals for the philosopher.

It is obvious that strict, formal, axiomatic systems, the only instances of mathematics according to the physicalist, have not always existed. Indeed, they had only been brought about in the wake of the foundation projects in the late nineteenth and early twentieth century. ${ }^{12}$ Even not drawing far-reaching conclusions from this fact, the physicalist must realize that the (only) objects

\footnotetext{
${ }^{10}$ Szabó (2010), p. 21.

${ }^{11}$ A label coined by Reuben Hersh: (contemporary) mathematicians, when asked of the nature of mathematics usually incline toward a formalist explanation while in their practice they work with their objects as "real" objects.

${ }^{12}$ Of course, their origins can be traced down in the works of Leibniz or even Euclid.
}

there are for mathematics (in his view) cannot be independent of human activity. Of course, he can say, human activity was indispensable for exhibiting some other systems in physics: (at least) instruments were needed to detect them. Now it would be of interest to show that the activity resulting in the exhibition of formal systems is by no means different from those giving us the possibility to talk about protons, neutrons and electrons, for instance. Or if it is different, showing its specific nature may have some morals. ${ }^{13}$ But the task does not seem to me trivial at all.

Moreover, historical consideration can shed some light on how does mathematics (in the usual sense of the word) really work. One can consult, for instance, the physicalist's only explanation for the existence of the actual mathematical objects: the alleged utilitarian factors behind the fact that some privileged systems are in the spotlight and not the infinitely many possible others - those only useful in physical theories. In the light of history, this assertion does not seem to be wholly convincing. True, there are theories whose births were inevitably urged by practical problems, e.g. analysis. But most of the theories are rather like non-Euclidean geometries: there were no practical constraints whatsoever as midwives at their births. And there is a bulk of mathematical theories whose practical applications are far from clear.

As for psychology, it exhibits time and again various patterns of pre-theoretic mathematical ability in adults, children or animals by strict, behavioristic means. Again, without going too far with the consequences, this could at least suggest that the actual systems dealt with in mathematical practice are not entirely arbitrary.

I will not go into details on these issues here. My intent was only to indicate that the position of the physicalist can be weakened by historical or psychological considerations (too). True, one can disregard these perspectives and put normative claims on mathematics. I will address this point soon. But let us stay with mathematical practice for a little more while now.

\section{Intuitions, Heuristic Do Not Belong to Mathematics}

It is a well-known fact that mathematical results reach far beyond human intuitions. Some say, there was a point somewhere around the birth of non-Euclidean geometries, when intuitions and modern mathematics divorced inevitably and for good and all. The situation is far not so black and white, however.

First, intuitive insight and "intended interpretations" can be blamed for those structures (axiomatic systems, if you like) that we regard as mathematics today, even if they had grown over human intuitions. It is not unthinkable that the physicalist would be ready to admit this point, only he would add as usual: this is a contingent "historical" fact.

Second, much depends on what we mean by intuition. We can insist that it is something like an eternal, unchangeable, mental vista which covers some areas of mathematics but not others. But we can be more permissive. If we let the concept to encompass any kind of, say, intuition-like heuristics, then we

\footnotetext{
${ }^{13}$ Since the physicalist regards formal systems as flesh and blood object, it seems to me he must accept that they are simply manmade - at least all of the symbols used for mathematics are such. Hence, one could think, maybe some "epistemology of artefacts" should be applied on them and methods akin to car testing or literary criticism...
} 
can confidently assert that intuition can be trained. In this case practicing mathematicians have much more intuition concerning complex structures than other earthborn beings.

Szabó (2010) declares that his physicalist account of mathematics concerns strictly the context of verification, not heuristics. In his opinion, creativity, intuition, and, what is more, human thought as such plays only a marginal role in the verifycation of mathematical propositions, for, essentially, proofs are nothing more than serials of formulas derived mechanically from the axioms. And these derivations often run far beyond the intuitive insight of a human. Nowadays, says Szabó, one cannot claim a proof to be surveyable, as it is clearly seen in the rather complex computer-based proof of the four-color theorem, for instance. ${ }^{14}$

First, it is worth to note in connection with the four-colour theorem, that the computer-based part of its proof is far not so complex. The thing is, that there are simply too many cases of a given (graph-theoretical) constellation-type for a human mathematician to check even along a lifetime. Yes, this part of the proof is mechanical, but not in the sense the physicalist would like to see. The (computerized, mathematical) system does not check what follows from the axioms of first-order logic along with the axioms of set theory and the definitions of graph theory, but it simply executes the algorithmic rules specified by the programmer (mathematician). The "rest" of the proof does require creativity and human thinking (including programming). And not necessarily in a weak sense: without them there would be no theorem whatsoever, (so there would be nothing to declare as a mere physical fact, as a mere consequence of some physical system).

On the other hand, some contemporary proofs like Wiles' on Fermat-conjecture ${ }^{15}$ are surveyable. True, maybe it is not surveyable for me, but it is for the professionals of the topic-or topics: the proof exploits numerous highly esoteric mathematiccal results. Anyway, I think that surveyability is a rather vague and, in addition, unimportant concept. The crucial thing lies elsewhere. It is hard to defend the claim, that a proof like the one by Wiles is nothing more than a mere mechanical derivation from the axioms. Indeed, it would be hard to model such a proof as a mechanical process. For one to build a computer program deriving the proof of Fermat-conjecture (say, in the style of Wiles), it seems to me, he must already know and understand the ideas applied in the proof given by Wiles!

Note that according to Szabó (2009) the "mechanism" of a formal system can be observed as a neurophysical process of the brain (Well, in a more correct wording: a serial of neurophysical processes in a brain accomplishing derivations is to be regarded as a genuine formal system). Note further that we cannot, certainly, talk about "representation" of a system, of a proof or something like that, because there is nothing to represent. There are only particular physical objects (and "physiccal” rules) remained for us as mathematics, ink-colored paper pulp, conducting wires, firing neurons. ${ }^{16}$ But as far as I know we are quite far from the understanding of brain processes, quite far from more or less justly regarding human brain as a Turing-machine (even though it is often regarded so despite of this fact), and very far from identifying the neurophysical process of adding five to seven. Though I understand that the formal sys-

\footnotetext{
${ }^{14}$ See Appel, Haken, Koch (1977) and Tymoczko (1979).

${ }^{15}$ Wiles (1995).

${ }^{16}$ Since these are the only things out there, they cannot be regarded as tokens not having types. I will address this rather problematical point in a moment.
}

tems thus revealed are of crucial importance for the physicalist, this area is so slippery that I think it is better to keep or attention on ink, paper and machines.

As far as I can see, the only plausible understanding of formal systems as physical phenomena is the following. Assume a Turing-machine with the axioms of logic, arithmetic, derivations rules etc. programmed in it. ${ }^{17}$ We can venture, that the machine will derive the proof of the Fermat-conjecture at some point we only have to wait long enough, since it will derive all of the theorems of the system if we wait infinitely long. Give a monkey a typewriter and infinite time, it will sooner or later put down the complete works of Shakespeare. ${ }^{18}$ So far so good, but what can we do with that fact? Can mathematics build on this result, and the infinite many other results so derived? Can they be grabbed, picked up or isolated? ${ }^{19}$ (And by whom?).

Sure, one can construct an arbitrary axiom system, since the adherence of mathematicians to the ones in use is another "contingent and unimportant” fact. Sure, she can translate it into algorithms to check the mechanical consequences by a Turing-machine. Sure, she will get arbitrarily long proofs/theorems (during an arbitrarily long time), which happen to be quite unsurveyable in every respect. But it seems also sure for me: maybe they can be seen as a genuine part of mathematics from some philosophical reconstruction, but they will be completely unimportant for and will have nothing to do with mathematics as a science. Mathematics as practice has also nothing to do with the bigger part of mathematics as so-labeled by the physiccalist.

\section{Gödel-Theorems and in General Meta-Mathematical Activities Do Not Belong to Mathematics}

Following Hilbert, the physicalist draws clear distinction between mathematics consisting of systems (somehow) built up by meaningless signs and activities dealing with these systems, namely meta-mathematics. The latter can make real meaningful statements about the formal systems (i.e. mathematics), which can be true (or false) in the semantic sense. In fact, meta-mathematical results are physical theories on physical formal systems expressed in some genuine language- - even though this language (or better: a part of it) highly resembles to the (meaningless) sign serials of the formal systems. It follows that the theorems of mathematical logic are to be confirmed by empirical means: by observations.

But what is the empirical content of Gödel-theorems, Löwenheim-Skolem-theorems, the theorem on the independence of continuum hypothesis etc.? Do they really assert a feature of a particular physical system? (Which one?) Maybe even the physicalist does not intend to claim that there is only one particular system the above theorems go for. On the other hand, it is quite implausible to say, that the confirmation goes like this: we pull out similar systems from a hat, one after other and check whether the given theorem holds in it or not. It is far from clear what feature of this meaningless serials of signs is

\footnotetext{
$\overline{{ }^{17} \text { But notice that going only that far is not at all unproblematic: at this point }}$ we have already much more than the mere axioms at play.

${ }^{18}$ Will it really, is another question. Could every finite sequence of formulas be enumerated by this "method", the monkey will die, the typewriter will go wrong in a (rather short) finite time. True, these are only "contingent facts of physics”...

${ }^{19}$ Of course, it is a contingent social fact for the physicalist whether a given theorem is of importance or not.
} 
asserted by the upper Löwenheim-Skolem-theorem, say. And it is far from clear how similarity is to be understood: my handwriting, a computer with an algorithm and some synaptic processes show very little similarity. As for those features which happen to be similar in these systems, I would be puzzled to show what Löweinheim-Skolem-theorem is to do with them.

\section{What, Then, Does Belong to Mathematics?}

A formal system is nothing more than axioms and rules, given as particular symbols, after all particular arrays of ink on a particular sheet. As it is a physical system, it must have some kind of behavior as, for instance, the solar system has, in which one can observe some regularities. What kind of "behavior" of this "system" can be identified by an observer not contaminated with platonic views from her schoolgirlhood-say, an alien? There is no such behavior, unless one does not mean the prima facie physical properties of the paper and the ink, which is probably not what the phyisicalist is up to. Because it must be seen, nothing mathematical follows from these physical properties.

For the strings of symbols to work as mathematics, the ink appearances must be realized as tokens. Thus, they must be realized by someone. But at this point we must postulate the inter-subjective existence of symbol types. Without recognising the types the given tokens belong to, one can identify exactly as many "systems" on an ink-marked sheet of paper as in a handful of ashes poured on the table.

\section{The First or the Last? (If at All...)}

Taking a glance at the history of mathematics it can be clearly seen, that philosophy and mathematics have always been living close, if you like, in a kind of symbiotic relationship. Also true, however, that the assessment of this relationship is quite far from being unanimous. Some scientists assert the primacy of philosophical considerations, claiming for clear philosophical foundations before beginning with mathematics. Errett Bishop, the father of constructive analysis was seemingly unsatisfied with the practice of his contemporary mathematiccian fellows:

"There is a crisis in contemporary mathematics. And anybody who has not noticed it is being wilfully blind. The crisis is due to our neglect of philosophical issues."20

But many maintain that philosophy has no legitimacy to lay down different norms for mathematics. As David Lewis writes:

"How would you like to go and tell the mathematicians that they must change their ways $[\cdots]$ ? Will you tell them, with a straight face, to follow philosophical argument wherever it leads? $[\cdots]$ [W]ill you boast of philosophy's $[\cdots]$ great discoveries: That motion is impossible [‥], that it is unthinkable that anything exists outside the mind, that time is unreal, that no theory has ever been made at all probable by evidence [‥]? Not me!"21

As often, the truth lies somewhere in the middle. Philosophy should and would not in itself determine the right terms for scientific practice and methodology. But it has the right to make critical observations on the methodologies of sciences or even normative recommendations. Let me give now an example outside of mathematics. Despite the fact that it is quite illegitimate to assume causal connection between phenomena in statistical

\footnotetext{
${ }^{20}$ Bishop (1975); recited in Shapiro (2000), p. 23.

${ }^{21}$ Lewis (1993); recited in Shapiro (2000), p. 30.
}

correlation, many sociologists do assume causal connection in those cases (Not to mention policy-makers). I think it is quite right to tell them they are wrong. On the other hand, they have the right not to change their well-tried ways with heavy traffic. Similarly, it is equally right to draw the mathematician's attention to the awkward consequences of the Axiom of Choice every now and then...

The physicalist view, as Szabó (2010) makes it clear, is an approach to mathematics following the philosophy-first principle (as opposed to the philosophy-last-if-at-all principle). ${ }^{22}$ As such, similarly to the intuitionist program, it prescribes for mathematics what it should be. Do not be misguided by the fact that it does it in a disguise of description. A description so far from the mathematics comprehended by common sense can be suspected to be a normative manifestation.

But the position of physicalism is much worse than that of intuitionism. The latter claims for serious methodological restrictions, and finds devoted followers among the practising mathematicians - while, of course, the majority adheres to the "Cantorian paradise". ${ }^{23}$ At the same time the physicalist, in aiming to show what mathematics really is, jettisons almost everything - including the whole methodology. For, according to this view, mathematical systems are nothing more than physical systems; there is no a priory knowledge; deduction is a special kind of induction; our only source of knowledge is empirical observation. And, to be sure, physics has its own methodology — why would another be needed?

Maybe the mathematician should be grateful to be so relieved from the methodological burden, but I have doubts about it. The situations is rather this: in trying to get rid of the all of the "verbal decoration" surrounding mathematics, the physicalist, it seems to me, gets rid of mathematics itself. Following him, the mathematician would be exiled not from the Cantorian but from the mathematical paradise.

\section{REFERENCES}

Appel, K., Haken, W., \& Koch, J. (1977). Every planar map is four colorable. Illinois Journal of Mathematics, 21, 439-567.

Ayer, A. J. (1936). Language, truth, and logic. London: Gollancz.

Bishop, E. (1975). The crises in contemporary mathematics. Historia Mathematica, 2, 505-517. doi:10.1016/0315-0860(75)90113-5

Gödel, K. (1944). Russell's mathematical logic. In P. Benacerraf, \& H. Putnam (Eds.), Philosophy of mathematics (2nd ed., pp. 447-469). Cambridge: Cambridge University Press.

Hilbert, D. (1926). Über das unendliche. Mathematische Annalen, 95, 161-190. doi:10.1007/BF01206605

Leacock, S. (1911). Gertrude the governess or simple seventeen. In: J. Lane (Ed.), Nonsense Novels (New ed.), London: New York Review Book.

Lewis, D. (1993). Mathematics is megethology. Philosophia Mathematica, 3, 3-23. doi:10.1093/philmat/1.1.3

Shapiro, S. (2000). Philosophy of mathematics. Oxford: Oxford Universit Press. doi:10.1093/0195139305.001.0001

Russell, B. (1912). The problems of philosophy. London: Williams and Norgate.

Szabó, L. E. (2003). Formal systems as physical objects: A physicalist account of mathematical truth. International Studies in the Philosophy of Science, 17, 117-125. doi:10.1080/0269859031000160568

Szabó, L. E. (2009). How can physics account for mathematical truth? Preprint, URL (last checked 26 January 2012). http://philsci-archive.pitt.edu/archive/00005338/

\footnotetext{
${ }^{22}$ A classification of Shapiro (2000)

${ }^{23}$ See: Hilbert (1926).
} 


\section{F. CSATÁRI}

Szabó, L. E. (2010). Lecture notes, philosophy of mathematics. Budapest: Eötvös Universty.

Tymoczko, T. (1979). The four-color problem and its philosophical significance. Journal of Philosophy, 76, 57-83. doi:10.2307/2025976 Wiles, A. (1995). Modular elliptic curves and Fermat's Last Theorem. Annals of Mathematics, 141, 443-551. doi:10.2307/2118559 